\title{
Impact of IEEE 802.15.4 communication settings on performance in asynchronous two way UWB ranging
}

\author{
Konstantin Mikhaylov' ${ }^{1}$ Juha Petäjäjärvi', Matti Hämäläinen ${ }^{1}$, Antti Tikanmäki ${ }^{2}$, \\ Ryuji Kohno \\ 'Centre for Wireless Communications, University of Oulu, Finland \\ \{firstname.lastname\} @ee.oulu.fi \\ ${ }^{2}$ Biomimetics and Intelligent Systems Group, University of Oulu, Finland \\ \{firstname.lastname\}@ee.oulu.fi \\ ${ }^{3}$ Division of Physics, Electrical and Computer Engineering, Yokohama National \\ University, Yokohama, Japan \\ \{lastname\}@ynu.ac.jp \\ ${ }^{4}$ University of Oulu Research Institute Japan CWC-Nippon Co. Ltd., Yokohama, \\ Japan
}

\begin{abstract}
The ultrawideband (UWB) radio signals are known for their good time resolution enabling implementation of accurate localization and tracking. The recent appearance of commercial UWB transceivers in masses on the market has boosted the interest towards this technology and facilitated its use not just for research, but also for business. In this paper we focus on the problem of UWB-based wireless indoor localization of machines and humans by means of IEEE 802.15.4-2015 high rate pulse repetition (HRP) UWB technology and specifically the accuracy of such localization. Namely, we report the results of an extensive experimental study revealing the effect of various communication settings on the accuracy of indoor localization for an asymmetric localization protocol. The conducted experiments lasted over 200 hours almost nonstop and involved transmission of more than 30 million ranging packets. In the experiments we have tested over 200 different modes and explored the effect of seven different parameters on the UWB ranging performance. The presented results reveal that the communication settings need to be accounted when determining the time of flight using UWB. Also we show that the accuracy of ranging is strongly affected by the used channel, data rate and pulse repetition frequency. Finally, we note that the increase of the UWB transceiver's temperature due to self-heating has a strong effect on the results of the localization.
\end{abstract}

Keywords: ultra wideband; IEEE 802.15.4; localization; asynchronous; ranging; localization; indoor; body area network; accuracy; experiment; measurements; performance; DW1000.

Acknowledgements: The research was undertaken in the context of the University of Oulu ITEE WIILMOR and EU FP7 CoNHealth projects. The work of the first author has been partially supported by the Nokia Foundation grants №201510090 and 201610150, and the Sähköinsinööriliiton Säätiö 2016 grant. Also the authors wish to thank Dr. Jussi Haapola from CWC for his advices and feedback about the composition of the planned experiments and the selection of parameters for them. 


\section{Introduction}

The problem of determining one's own position in space was one of the fundamental challenges for humanity since its very early days. The technological breakthroughs of the $20^{\text {th }}$ century have enabled the development of the global navigation satellite system (GNSS) technologies, which are seen as a decisive step on the way to ultimately solve this challenge. Not surprising, at the present day a navigation module capable of getting the signals of one or even several GNSSs became a critical component of any contemporary smart phone, thus enabling its user to determine his place anywhere on Earth, where the direct line-of-sight (LoS) for several satellites is available. Although area of the locations where the stated above condition is not valid and where the GNSSs are not available may seem to be not very high overall, many of the urban territories (e.g., the "urban canyons") as well as the indoor locations, which are the places where many people spend most of their time, are among them. To give a real life example, as this was reported by B.R. de Castro et al. in [1], the median time spent by an average high school student outdoors, where there are good chances to catch a signal from a GNSS, is well below 2 hours a day. Even though the localization indoors may not be of such a high demand as the localization outdoors, its unavailability can become a substantial problem. What is more than this, the long-talked-about concept of the Internet of Things (IoT) presumes that a number of the electronic devices around us in the future will drastically increase. Many of these devices will accompany us $24 \times 7$ thus becoming an important component of our body area networks (BANs). For them, the information about their current context, which is inevitably linked to their location, is also of extreme importance.

These two simple motives illustrate the importance of enabling the accurate indoor localization and explain why over the recent years this problem has been approached from various angles and the solutions based on the different principles have been proposed and investigated. It has been shown [2,3] that the indoor localization can be accomplished by the means of acoustic, ultrasonic, radio frequency (RF), light and infrared-based signals. The position can also be determined by measuring the variety of the environment factors like, the strength of electro-magnetic field, the level of light or noise. Next, given that the initial position is known, the inertial navigation based on the data about the velocities and orientation is feasible. Finally, the localization can be accomplished by the means of machine-vision techniques [4].

Among all these diverse localization methods the RF-based solutions were traditionally getting a lot of attention. The great number of the device-based and device-free [5] solutions based on the various standards (IEEE 802.11, IEEE 802.15.4, RFID, Bluetooth, GSM are among them) and proper solutions have been proposed and investigated. Among these, the systems based on the use of ultra wideband (UWB) 
signals are of especial interest, since this technology offers the potential of achieving high ranging accuracy due to its good time domain resolution even in harsh indoor environments [6,7]. Even though this technology has been on the table for almost two decades now, it was not until few years ago when the real commercial UWB transceivers started to appear on the market in mass scales. This brought more interest towards the UWB technology and boosted the use of the UWB based localization solutions both by academy ${ }^{1}$ and business alike. The use of this technology in BANs and the different human-centric applications is especially attractive.

In the current paper we investigate the effect of the various communication settings and localization protocol parameters on the accuracy of wireless indoor localization accomplished with an single-sided asymmetric two way ranging localization protocol. The results of an extensive real-life measurement campaign conducted with the hardware IEEE 802.15.4-2015 high rate pulse repetition (HRP) compatible UWB radio transceivers are reported and analyzed in this paper. The presented results capture several important dependences in respect to the accuracy of UWB-based indoor localization, thus enabling a justified selection of the communication and protocol parameters maximizing the precision of UWB-based localization.

The paper is organized as follows. First, in Section 2, we address some of the previous works in the field. In Section 3 we highlight the important specifics of the IEEE 802.15.4-2015 HRP UWB-based communication and localization. Section 4 describes the results of the conducted measurement campaign and the analysis of these results. Finally, Section 5 concludes the paper and summarizes the lessons learned.

\section{$2 \quad$ Related works}

The amount of the literature where the authors focus on the various aspects of designing and using the RF-based indoor localization systems in general and the UWB ones in particular is really tremendous. For the overview of the different approaches and problems related to them, the reader can check, e.g., [3],[6],[7],[9]. Given the enormous number of the papers, in what follows we specifically focus on the papers addressing

${ }^{1}$ A good illustration for this is the fact that over a half of the solutions presented in the Microsoft Indoor Localization Competition in 2016 were based on UWB [8]. 


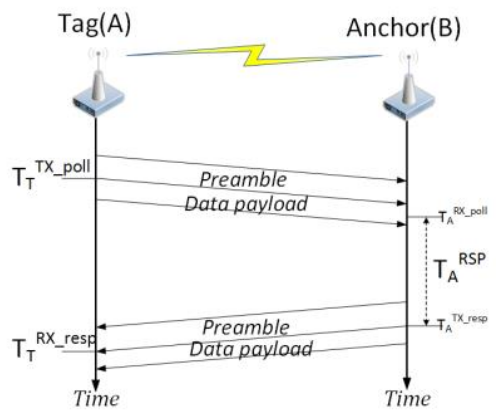

(a) basic TWR procedure

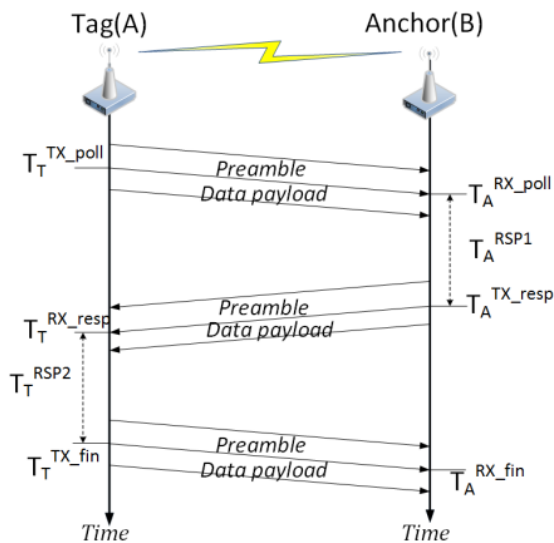

(b) symmetric double-sided TWR procedure

Fig. 1. Traditional ranging protocols for UWB localization.

the two-way UWB localization protocols based on round trip time estimation (RTT) on one hand and the studies revealing the effect of the communication parameters on the UWB performance on the other.

As discussed in [10] in the absence of the synchronization between the two devices (A and B) the RTT can be estimated in three ways. The first option is based on a transmission of a packet from node A to node B followed by a packet sent from node B to node A (depicted in Fig. 1 (a)). This method is referred to as "basic TWR" further in this paper and for it the time-of-flight (TOF) is calculated with

$$
T O F_{T W R}=0.5\left(\left(T_{T}^{R X_{-} \text {resp }}-T_{T}^{T X_{-} \text {poll }}\right)-\left(T_{A}^{T X_{-} \text {resp }}-T_{A}^{R X_{-} \text {poll }}\right)\right)
$$

where $T_{x}^{y}$ designate the timestamps detailed in Fig. 1 (a). The critical problem for this solution is the clock drift [10]. The second option is the Symmetric Double-Sided TWR (SDS-TWR) protocol (depicted in Fig. 1 (b)) designed by Nanotron [11] and adopted by IEEE 802.15.4-2015 standard [12]. The TOF for SDS-TWR is calculated based on

$$
\begin{aligned}
T O F_{S D S-T W R}=0.25 & \left(\left(T_{T}^{R X_{-} \text {resp }}-T_{T}^{T X_{-} \text {poll }}\right)-\left(T_{A}^{T X_{\text {resp }}}-T_{A}^{R X_{\text {poll }}}\right)\right. \\
& \left.+\left(T_{A}^{R X_{-} f i n}-T_{A}^{T X_{-} r e s p}\right)-\left(T_{T}^{R X_{-} f i n}-T_{T}^{T X_{-} r e s p}\right)\right)
\end{aligned}
$$

with $T_{x}^{y}$ detailed in Fig. 1 (b). This method can effectively mitigate the clock drift problem. As this was shown in [13], the performance of the SDS-TWR can be further improved if instead of one reply node B will send multiple. This protocol got the name of SDS-TWR with Multiple Acknowledgement (SDS-TWR-MA). Another variation of this protocol (Double Two-Way Ranging (D-TWR)) has been proposed by the authors in [14], where the number of requests from node A was multiplied. In [15] and [16] the modifications for the SDS-TWR for enabling more efficient operation in multi-anchor environment were proposed. The results of the practical evaluation of these protocols 
and their comparison with SRS-TWR using the commercial UWB transceivers from Decawave are reported in [17] showing that the mean error with SDS-TWR is in the order of $30 \mathrm{~cm}$ and for the proposed protocols - from 40 to almost $100 \mathrm{~cm}$. Finally, the authors of [18] presented the PolyPoint localization system and the respective localization protocol exploiting both frequency and antenna diversity. One of the common things for all the discussed protocols is the fact that all of them are symmetric in a sense that the time stamping is executed by both nodes and the RTT estimation is done by combining the measurements coming from both nodes.

Even though the performance and the effect of the various communication mode parameters on the performance of the UWB communication have been addressed in multiple studies, none of the works known by the authors provides a comprehensive picture. E.g., in [19] and [20] the authors analytically characterized the effect of the rate, the pulse repetition frequency (PRF) and the preamble length on the packet's on-air time and maximum throughput. The effect of the preamble length and the channel used on the communication distance was analytically studied in [21]. The effect of the symbol rate on the bit error rate for UWB assuming different receiver architectures was simulated in [22]. The results of the experiments carried using the UWB transceivers were reported, e.g., in [23]-[27]. Nonetheless, in all these works the authors have characterized the accuracy of localization only for one particular operation mode of the UWB transceiver. The three different operating modes were evaluated by the authors in [28]. The results of experimental evaluation of the effect of some communication mode parameters on the communication range and packet error rate for IEEE 802.15.4a compatible transceiver were reported in [29].

\section{IEEE 802.15.4 UWB based communication and ranging.}

As discussed, e.g., in [6], the impulse radio UWB communication systems use very short, nano- or picosecond-long, pulses with ultra-wide spectral requirement in frequency domain (i.e., having signal bandwidth over $500 \mathrm{MHz}$ ). Although there are a few different ways how these pulses may be modulated with data, one of the most common approaches is to utilize the pulse position modulation (PPM) [6]. Typically, a single transmitted symbol is spread over multiple pulses (or pulse bursts) in order to achieve processing gain. To give a practical example, in IEEE 802.15.4-2015 standard [12] (following the IEEE 802.15.4a[30] and IEEE 802.15.4-2011[31] standards), the HRP UWB waveform during $k$-th symbol is given by:

$$
x^{k}(t)=\left[1-2 g_{1}^{(k)}\right] \Sigma_{n=1}^{N_{c p b}}\left[1-2 s_{n+k N_{c p b}}\right] \times p\left(t-g_{0}^{(k)} T_{B P M}-h^{(k)} T_{\text {burst }}-n T_{c}\right),
$$

where $g_{0}^{(k)}$ and $g_{1}^{(k)}$ are the bits encoded by position and polarity of the burst, respectively, $N_{c p b}$ is the number of pulses in a burst, $s_{n+k N_{c p b}}$ is the scrambling code 
used, $p(t)$ is the shape of the transmitted symbol, $T_{c}$ is a duration of a possible chip position, $T_{B P M}$ is the burst position modulation (BPM) interval, $T_{\text {burst }}$ is the duration of a burst and $h^{(k)}$ is the burst hopping sequence. As can be seen from Fig. 2, an UWB pulse (burst) occupies only a portion of the entire symbol period. The two major consequences of this are the low duty cycle of a UWB transmission, which reduces the total consumed energy, and the tolerance of UWB signals to multipath propagation, which is characteristic for the indoor environment. Another feature enabled by the use of extremely short pulses is a very good time domain resolution for radio signal arrival estimation in location and tracking applications [6].
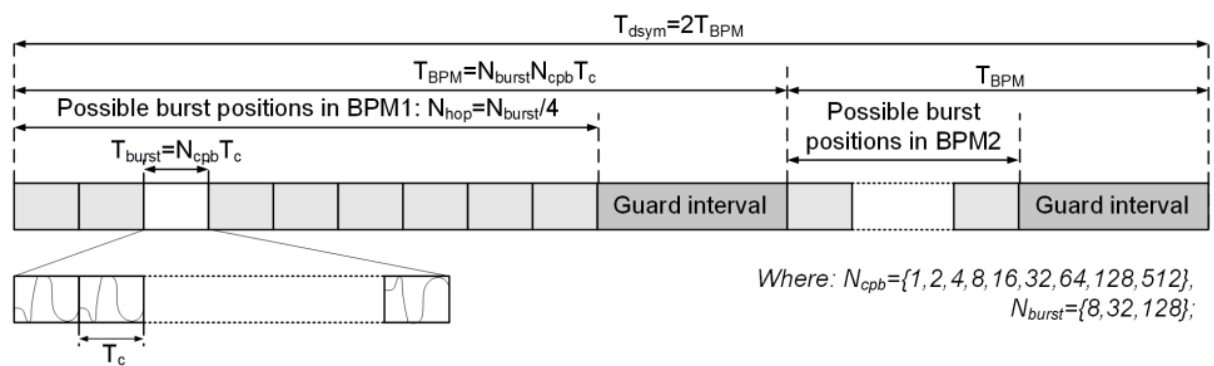

Where: $N_{c p b}=\{1,2,4,8,16,32,64,128,512\}$ $N_{\text {burst }}=\{8,32,128\}$.

Fig. 2. IEEE 802.15.4-2015 HRP UWB symbol and respective timings.

The format of a physical layer (PHY) protocol data unit (PPDU) prescribed by IEEE 802.15.4 for UWB is depicted in Fig. 3. A frame starts with the synchronization header (SHR), containing two components. The former one is the SYNC field, which is used for automatic gain control (AGC) convergence, diversity selection, timing acquisition, and coarse frequency acquisition [12]. The latter is the start-of-frame delimiter (SFD) indicating the start of data portion. The SYNC portion is composed of $16,64,1024$, or 4096 repetitions of a preamble symbol, which is obtained by spreading one of the 24 possible preamble codes with perfect periodic autocorrelation properties. The length of a code may be set at either 31 or 127, and the lists of the available options vary for different operating channels. The duration of the SFD differs depending on the data rate used for the data field and equals either 8 or 64 preamble symbols. The SHR is followed by the PHY header (PHR), which consists of 19 bits and conveys information necessary for a successful decoding of the packet, including the PHY service data unit (PSDU) data rate, preamble duration, and the length of the frame payload [12]. The field following the PHR contains the data encoded using the Reed-Solomon block code, which are further spread and modulated using BPM-BPSK modulation given by (3). 


\begin{tabular}{|c|c|c|c|}
\hline \multicolumn{2}{|c|}{$\begin{array}{c}\text { SHR preamble } \\
\text { (coded at base rate) }\end{array}$} & \multirow{2}{*}{$\begin{array}{l}\text { PHY header (PHR), } \\
\text { BPM-BPSK coded } \\
\text { at } 110 \text { or } 850 \mathrm{kbit} / \mathrm{s}\end{array}$} & \multirow{2}{*}{$\begin{array}{l}\text { Data field, BPM- } \\
\text { BPSK coded at rate } \\
\text { indicated in PHR }\end{array}$} \\
\hline $\begin{array}{c}\text { SYNC } \\
(16,64,1024 \text { or } 4096 \text { symb }) \\
\end{array}$ & $\begin{array}{l}\text { SFD } \\
\text { (8 or } 64 \text { symb) }\end{array}$ & & \\
\hline
\end{tabular}

Fig. 3. IEEE 802.15.4 PPDU.

Support of ranging for the UWB-enabled IEEE 802.15.4 devices is optional [12]. Albeit there is no reason preventing use of the whole sheer diversity of the different RF localization techniques developed over the past years (like, the ones based on radio signal strength indicator (RSSI)) with UWB, the good time domain resolution of the UWB signals encourages using the techniques based on TOF estimation. Based on the application's requirements and the specifics of the operation environment any of the three basic TOF techniques can be employed [7], i.e., the one-way time-of-arrival (TOA), the two-way TOA, or the time-difference-of-arrival (TDOA). The first and the last approaches require a mechanism (typically implemented based on wired communication) for accurate synchronization of the clocks on the nodes, whilst the second option eliminates the synchronization error in the process of TOF measurements. All the protocols discussed in the previous section belong to two-way TOA.

To enable ranging the IEEE 802.15.4 prescribes the ranging-capable devices (RDEVs) to implement a special ranging counter operating at $1 / 128$ of a chip time at the chip rate of $499.2 \mathrm{MHz}$. Additionally, for every ranging counter value that is produced by RDEV the transceiver should generate the figure of merit characterizing the accuracy of the estimate of the arrival time of the leading edge of the first pulse of the packet header at the antenna. Depending on the scenario and the targeted application, ranging can be done, e.g., by two-way ToF measurement, or using one way and an infrastructure of RDEV with common notion of time [12]. Note, that the IEEE 802.15.4 ranging frames can carry data and differ from the other packets only in the ranging bit set in their PHR.

As this has been discussed in [7], there are three major sources of errors for ToF-based UWB ranging. The first one is the effect of radio wave propagation and of the path taken by a radio signal. The multipath fading, the excess delay and pulse shape distortion for propagating through various obstacles and the blockade of the direct path can strongly affect the obtained result. The second possible issue is the interference caused by other narrow-band or wide-band wireless systems. Finally, the third problem is the non-ideality of the hardware and of the restrictions of the employed technical solutions. To give a few examples: the drift of the clock, the limited sampling rate for the time stamp, and the variation of the on-board propagation path for different radios can introduce an additional error in the ranging measurements. 
Another aspect which can affect the accuracy of ranging is the choice of the UWB communication parameters. As easy to see from (3) and Figs. 2 and 3, there are multiple variables affecting the UWB frame composition and signal shape which can potentially affect the ranging accuracy, including: the shape and duration of a pulse, number of pulses in a burst, duration of the interval between the bursts, operating channel, preamble duration and the used preamble code, SFD duration, etc. Analytically, effect of some of these parameters for various UWB architectures was investigated e.g., in [32], [33] and [34].

\section{Experimental evaluation of the effect of UWB communication parameters on the performance of wireless indoor localization}

In [35] we have presented the single-sided asymmetric TWR (SSA-TWR) protocol and reported the results of its extensive evaluation, assessing the feasibility and efficiency of the proposed protocol. Also these measurements revealed the strong effect of the various communication parameters on the accuracy of UWB localization for the tested protocol as well as for the basic TWR and SDS-TWR protocols, which were used as the references for comparison. Motivated by this observation, we expanded our experiments in order to investigate how the various communication mode parameters affect the results of localization in real-life scenarios. In the following sections we first explain our experimental set up and the used test procedure, and then present and comment on some of the obtained results.

\subsection{Experiment set up and the tested modes}

In the tests we used the modular plug\&play sensor network and IoT platform reported in [36]. The platform enables construction of the nodes with the desired hardware architecture by stacking one or multiple various hardware modules (i.e., shields) on top of a single main board hosting the main processing unit (MPU), the power regulating circuitry and a set of basic interfaces. All the attached modules are automatically detected and identified by the MPU (using the procedure detailed in [37]) and the interfaces enabling the direct communication between the available on the modules peripherals and the MPU are automatically mapped. For this study we have used the first version of the main boards, which are built around the STM32F207 32-bit microcontroller. To enable the UWB communication, an UWB module based on the IEEE 802.15.4-compatible DWM1000 radio module [38] was attached to the main board. Additionally, the mobile tag node was equipped with an USB-UART interface module. For ensuring the sustainable power supply during the experiments, all nodes were powered from mains using an AC-DC converter and an USB cable. The hardware 
used for testing is illustrated in Fig. 4. It is important to note that since all the nodes were equipped with identical UWB hardware transceivers, the characteristics of their clocks are expected to be similar.

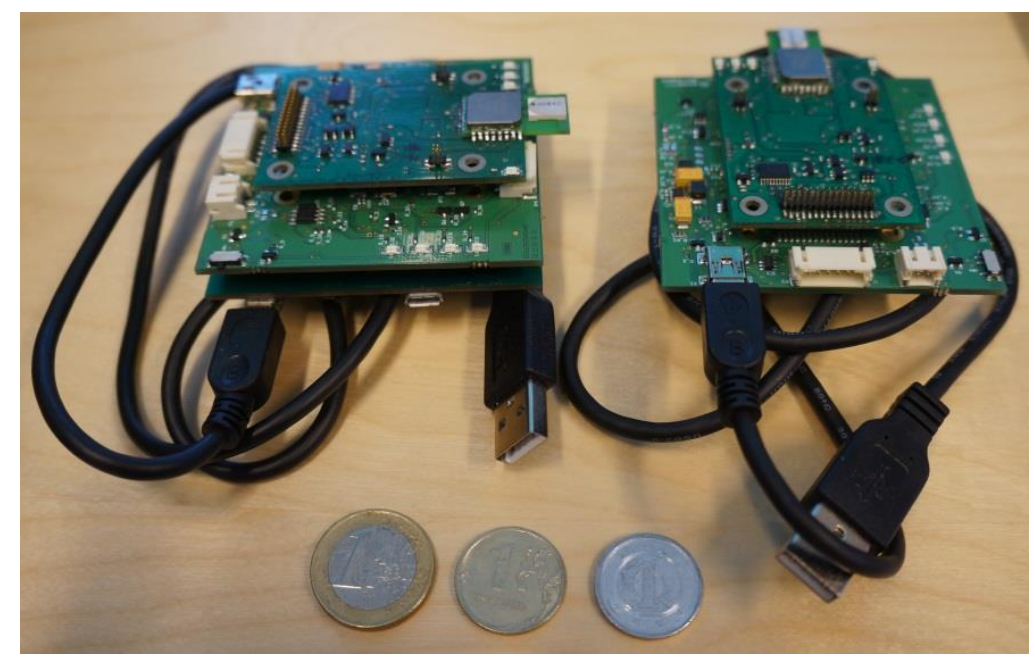

Fig. 4. Illustration of the hardware used for the tests (the tag on the left, an anchor on the right).

The nodes were programmed with the in-house developed software composed of the UWB transceiver drivers and the modified software implementing the SSA-TWR protocol. The original SSA-TWR software [35] was modified to enable support for more anchors and to handle the automatic switching of the modes. Also a special Javabased application has been developed for a laptop to automatize the mode switching and data logging. The state machines for the Java application, tag and anchor nodes, and the test procedure are depicted in Figs. 5 (a)-(d).The experiments were carried out in the indoor environment depicted in Fig. 6, using the three nodes: one tag (node A) and two anchors (nodes $\mathrm{C}$ and $\mathrm{D}$ ).

The procedure of the test majorly repeated the one used in [35]. The nodes were placed on the tripods having the height of $1.35 \mathrm{~m}$ ( $\mathrm{tag}$ ) and $1.05 \mathrm{~m}$ (anchors). The distance between the tag and the anchor nodes was controlled using a laser distance meter with the accuracy of $0.5 \mathrm{~cm}$ using the centers of UWB module's antennas as the points of reference. The newly introduced anchor node $(C)$ was mounted on a $1.05 \mathrm{~m}$ high tripod and was kept static at a distance of 0.85 meters from the tag. The reason after adding the third node was threefold. First, these results are intended to be used as the references for validation purposes. Second, they show the effect of the $T_{A}{ }^{R S P}$. Third, they give a hint for how the results change between the individual devices. The measurements were conducted for the 5 different distances between the tag and the anchor (D). At each of 
these points 5000 ranging experiments (procedure depicted in Fig. 5(d)) for each of the 36 calibration and 216 different test modes were completed sequentially.

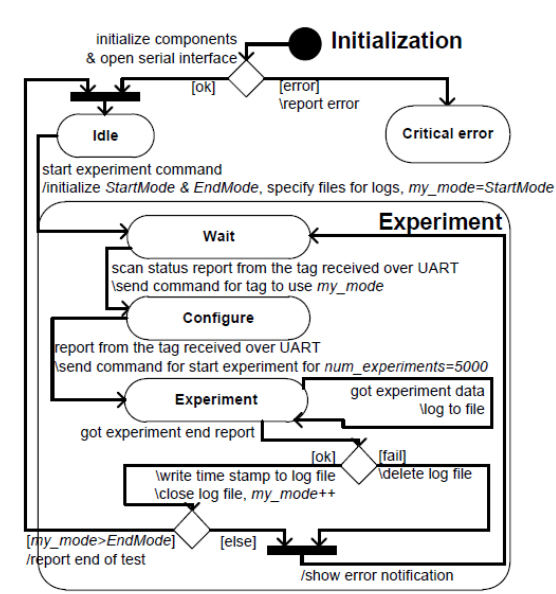

(a) state machine for Java application

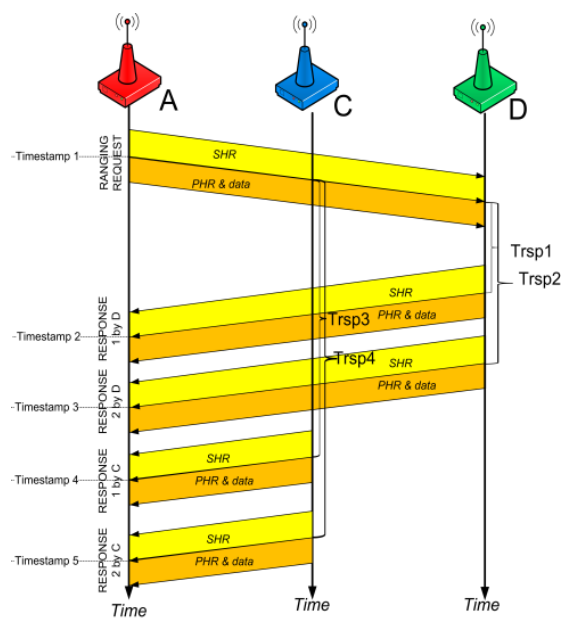

(d) procedure for a single ranging experiment

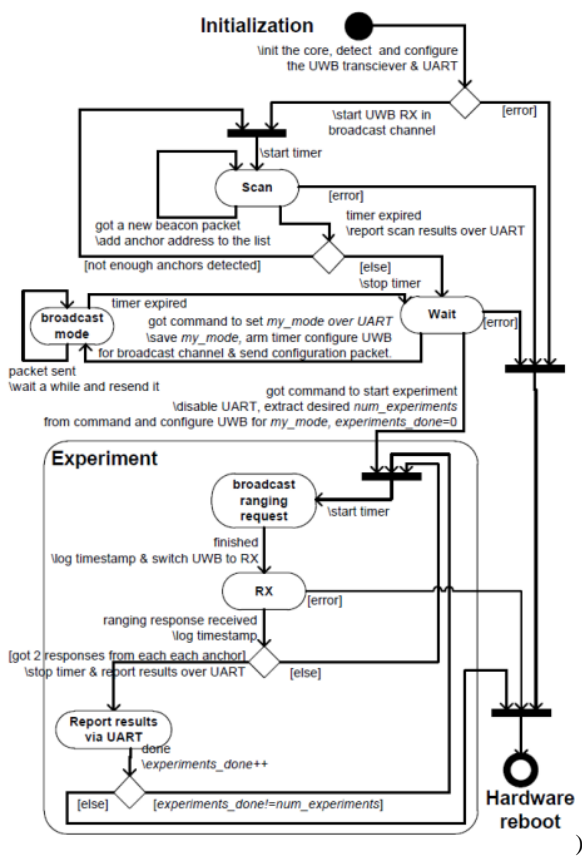

(b) state machine for the tag

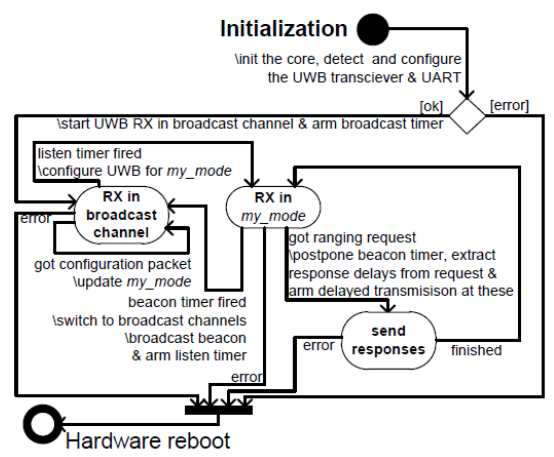

(c) state machine for an anchor

Fig. 5. State machines and the procedure for a single ranging experiment ${ }^{2}$

\footnotetext{
2 The following settings were used during the experiments: $T_{\text {rsp } 1}=T_{A}{ }^{R S P}, T_{r s p 2}=2 T_{A}{ }^{R S P}$, $\mathrm{T}_{\mathrm{rsp} 3}=3 \mathrm{~T}_{\mathrm{A}}^{\mathrm{RSP}}, \mathrm{T}_{\mathrm{rsp} 4}=4 \mathrm{~T}_{\mathrm{A}}^{\mathrm{RSP}}$
} 
The experiments lasted almost non-stop for over 200 hours and included transmission of over 30 millions ranging packets. The time stamps for each transmitted and receive by the tag packets as well as the other relevant data were logged down during the experiment. The results were first stored in the memory of the laptop and then statistically processed. For estimating the basic TWR the two packets of SSA-TWR were used. The UWB transceivers were configured to operate using the maximum power allowed (i.e., corresponding to $-41 \mathrm{dBm}$ in each $1 \mathrm{MHz}$ bandwidth).

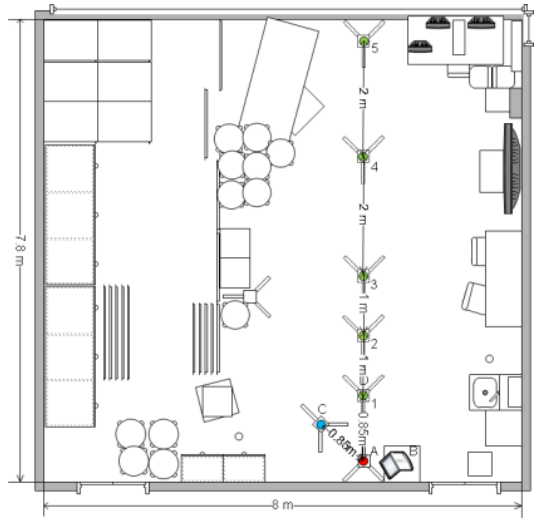

(a) plan of the room and test points

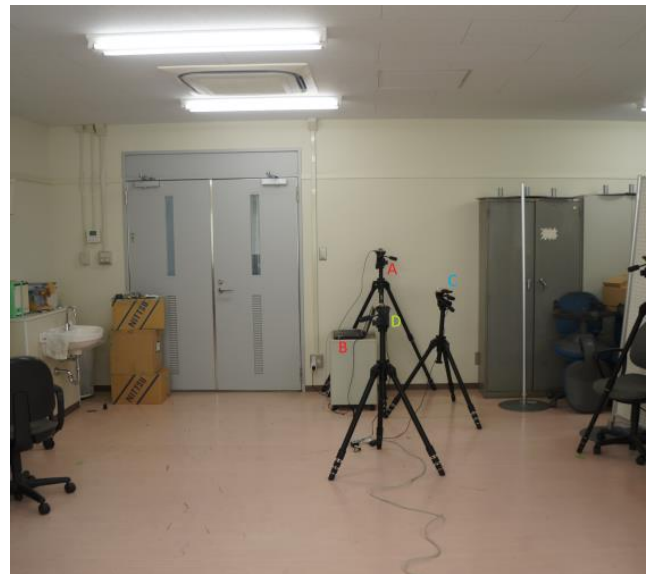

(b) foto of the set up (A - tag, B - laptop connected to tag, C and D - anchor nodes)

Fig. 6. Experiment environment

The composition and the sequence of test modes for one particular position of node $\mathrm{D}$ are detailed in Fig. 7. As can be seen from Fig. 7 (a), each test is divided into calibration and experimental blocks. The former ones are used for validating the results and are launched after testing each new 72-mode block. The configurations used for the calibration experiments are detailed in Fig. 7 (b). The configurations for the experimental blocks were selected to enable validation of the effects of all the major UWB communication settings. Namely, the different modes vary in terms of the used channels ( 3 options), number of the symbols in packet's preamble ( 3 options), the code ( 2 options for pulse repetition frequency (PRF) of $16 \mathrm{MHz}, 4$ options for PRF=64 MHz), PRF (2 options), the preamble acquisition chunk (PAC) size and the data rate. The tested channels were selected to enable evaluating the effects of both the difference in the central frequency (channels 2 and 5) and the bandwidth (channels 2 and 4). For the preamble length we have used long, middle and short preambles. The reason for not using the preambles shorter than 256 symbols is the weaker stability of the communication for these modes (e.g., refer to [29]). For PAC we have checked both a small value (i.e., 8) and a value recommended by the manufacturer of the transceiver 


\begin{tabular}{|l|l|}
\hline ID & Clarification \\
\hline V0-v8 & Calibration block1 \\
\hline 1 -36 & Experiment block (Channel 2, 110 kbit/s) \\
\hline $37-72$ & Experiment block (Channel 2,850 kbit/s) \\
\hline v9-v17 & Calibration block2 \\
\hline $73-108$ & Experiment block (Channel 4, $110 \mathrm{kbit} / \mathrm{s}$ ) \\
\hline $109-144$ & Experiment block (Channel 4,850 kbit/s) \\
\hline V18-V26 & Calibration block3 \\
\hline $145-180$ & Experiment block (Channel 5, 110 kbit/s) \\
\hline $181-216$ & Experiment block (Channel 5,850 kbit/s) \\
\hline V27-v35 & Calibration block4 \\
\hline
\end{tabular}

(a) sequential order of a test run for a single reference point

\begin{tabular}{|c|c|c|c|c|c|c|c|}
\hline ID & Channel & $\begin{array}{c}\text { Preamble, } \\
\text { symb }\end{array}$ & $\begin{array}{c}\text { Preamble } \\
\text { code }\end{array}$ & PAC & PRF & $\begin{array}{c}\text { Rate, } \\
\text { kbps }\end{array}$ & $\begin{array}{c}T_{A}{ }^{\text {RSP }} \\
\text { ms }\end{array}$ \\
\hline $\mathrm{V}(0+9 k)$ & 4 & 1024 & 1 & 32 & 64 & 850 & 15 \\
\hline $\mathrm{V}(1+9 k)$ & 4 & 1024 & 1 & 32 & 64 & 850 & 5 \\
\hline $\mathrm{V}(2+9 k)$ & 4 & 1024 & 1 & 32 & 64 & 850 & 15 \\
\hline $\mathrm{V}(3+9 k)$ & 4 & 1024 & 1 & 32 & 64 & 850 & 8 \\
\hline $\mathrm{V}(4+9 k)$ & 4 & 1024 & 1 & 32 & 64 & 850 & 15 \\
\hline $\mathrm{V}(5+9 k)$ & 4 & 1024 & 1 & 32 & 64 & 850 & 12 \\
\hline $\mathrm{V}(6+9 k)$ & 4 & 1024 & 1 & 32 & 64 & 850 & 15 \\
\hline $\mathrm{V}(7+9 k)$ & 4 & 1024 & 1 & 32 & 64 & 850 & 18 \\
\hline $\mathrm{V}(8+9 k)$ & 4 & 1024 & 1 & 32 & 64 & 850 & 15 \\
\hline & & & & & & & \\
\hline
\end{tabular}

(b) setting for test modes in calibration blocks

\begin{tabular}{|c|c|c|c|c|c|c|c|c|c|}
\hline ID & $\begin{array}{c}\text { Preamble, } \\
\text { symb }\end{array}$ & $\begin{array}{c}\text { Preamble } \\
\text { code }\end{array}$ & PAC & PRF & ID & $\begin{array}{l}\text { Preamble, } \\
\text { symb }\end{array}$ & $\begin{array}{c}\text { Preamble } \\
\text { code }\end{array}$ & PAC & PRF \\
\hline $1+36 \mathrm{k}$ & 256 & 1 & 8 & 16 & $19+36 \mathrm{k}$ & 256 & 2 & 8 & 64 \\
\hline $2+36 \mathrm{k}$ & 256 & 1 & 16 & 16 & $20+36 \mathrm{k}$ & 256 & 2 & 16 & 64 \\
\hline $3+36 \mathrm{k}$ & 1024 & 1 & 8 & 16 & $21+36 \mathrm{k}$ & 1024 & 2 & 8 & 64 \\
\hline $4+36 \mathrm{k}$ & 1024 & 1 & 32 & 16 & $22+36 \mathrm{k}$ & 1024 & 2 & 32 & 64 \\
\hline $5+36 k$ & 4096 & 1 & 8 & 16 & $23+36 \mathrm{k}$ & 4096 & 2 & 8 & 64 \\
\hline $6+36 k$ & 4096 & 1 & 64 & 16 & $24+36 k$ & 4096 & 2 & 64 & 64 \\
\hline $7+36 k$ & 256 & 2 & 8 & 16 & $25+36 \mathrm{k}$ & 256 & 3 & 8 & 64 \\
\hline $8+36 \mathrm{k}$ & 256 & 2 & 16 & 16 & $26+36 k$ & 256 & 3 & 16 & 64 \\
\hline $9+36 \mathrm{k}$ & 1024 & 2 & 8 & 16 & $27+36 \mathrm{k}$ & 1024 & 3 & 8 & 64 \\
\hline $10+36 \mathrm{k}$ & 1024 & 2 & 32 & 16 & $28+36 \mathrm{k}$ & 1024 & 3 & 32 & 64 \\
\hline $11+36 \mathrm{k}$ & 4096 & 2 & 8 & 16 & $29+36 \mathrm{k}$ & 4096 & 3 & 8 & 64 \\
\hline $12+36 \mathrm{k}$ & 4096 & 2 & 64 & 16 & $30+36 \mathrm{k}$ & 4096 & 3 & 64 & 64 \\
\hline $13+36 \mathrm{k}$ & 256 & 1 & 8 & 64 & $31+36 \mathrm{k}$ & 256 & 4 & 8 & 64 \\
\hline $14+36 k$ & 256 & 1 & 16 & 64 & $32+36 k$ & 256 & 4 & 16 & 64 \\
\hline $15+36 k$ & 1024 & 1 & 8 & 64 & $33+36 k$ & 1024 & 4 & 8 & 64 \\
\hline $16+36 k$ & 1024 & 1 & 32 & 64 & $34+36 \mathrm{k}$ & 1024 & 4 & 32 & 64 \\
\hline $17+36 k$ & 4096 & 1 & 8 & 64 & $35+36 \mathrm{k}$ & 4096 & 4 & 8 & 64 \\
\hline $18+36 \mathrm{k}$ & 4096 & 1 & 64 & 64 & $36+36 \mathrm{k}$ & 4096 & 4 & 64 & 64 \\
\hline
\end{tabular}

(c) settings for test modes in experiment blocks

Fig. 7. Tested modes

for the used preamble length. For PRF and preamble codes, all the options supported by the transceiver have been tested. Note that for all the modes in the experiment block, the $\mathrm{T}_{\mathrm{A}}{ }^{\mathrm{RSP}}$ was fixed at $15 \mathrm{~ms}$. Given the proven strong effect of the $\mathrm{T}_{\mathrm{A}}{ }^{\mathrm{RSP}}$ on the accuracy (refer to [35]), motivation of this choice may not be obvious. The reason for setting this value is that in order to characterize the effect of the communication parameters, all the modes should be tested with the same $\mathrm{T}_{\mathrm{A}}{ }^{\mathrm{RSP}}$ value. Meanwhile, the transmission of a single test packet with 4096 symbol preamble and 110 kbps data rate can take over 10 ms. Thus, accounting for the microcontroller-transceiver serial communication and the processing overhead, we specified $\mathrm{T}_{\mathrm{A}}{ }^{\mathrm{RSP}}=15 \mathrm{~ms}$. 


\subsection{Analysis of results for calibration runs.}

The logged experimental data have been further processed to calculate the mean RTT and its standard deviation for the different modes for all the nodes. The results were analyzed for the two cases, namely the basic TWR ranging (using either the first response packet (labeled as RTT1) or the second one (labeled as RTT2)) and for SSATWR operation.

First of all, to assess the validity of all the measurement results we have analyzed and compared the results for all calibration measurements. Figs. 8 (a)-(c) show the distribution of the mean RTT values for $1^{\text {st }}, 3^{\text {rd }}, 5^{\text {th }}, 7^{\text {th }}$ and $9^{\text {th }}$ experiments $\left(\mathrm{T}_{\mathrm{A}}{ }^{\mathrm{RSP}}=15\right.$ $\mathrm{ms}$ ) in all calibration blocks throughout the experiments. As can be seen, there are no anomalies; for the mobile node, the value of the RTT increases with the increase of the distance; for the static nodes throughout all the experiments the reported RTT remains the same. It can be clearly seen that the deviation of the values for the RTT for SSATWR is much lower than the one for basic TWR (the chart for SSA-TWR for the static anchor is not shown, but it has the same trend). The deviation of the RTT measurements for the node $\mathrm{C}$ is much higher than the one for node $\mathrm{D}$, which happens due to the different response delays for the nodes. Additionally, Fig 8 (d) illustrates the effect of the $\mathrm{T}_{\mathrm{A}}{ }^{\mathrm{RSP}}$ on the measured RTT (for node C and SSA-TWR the similar behavior was observed). The observed results correlate with the ones reported in [35]. Comparison of the Figs. 8 (a) and (c) shows that even when the two nodes were placed at about the same distance $(0.85 \mathrm{~m})$ the measured

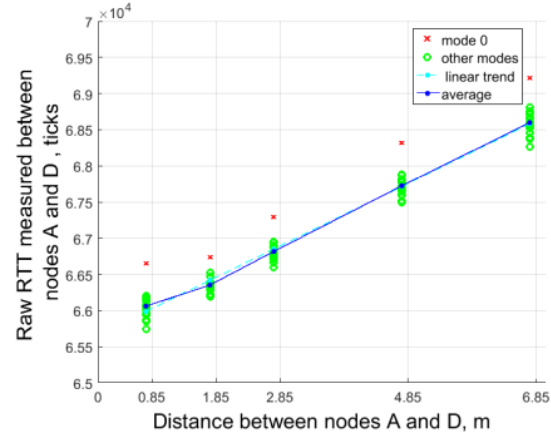

(a) mean RTT for node D measured with basic TWR (RTT1)

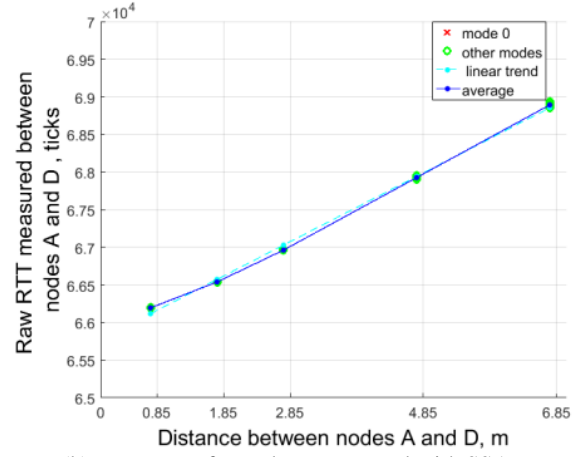

(b) mean RTT for node D measured with SSA-TWR 


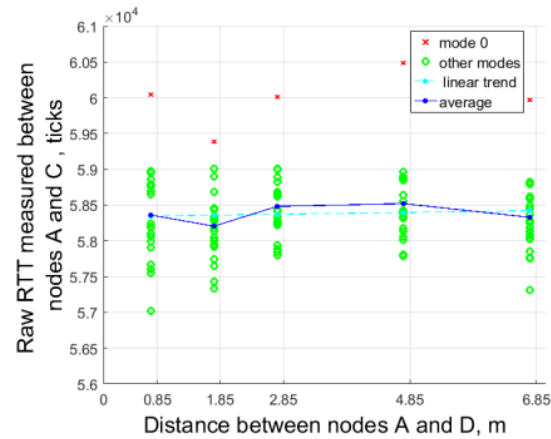

(c) mean RTT for node C measured with basic TWR (RTT1)

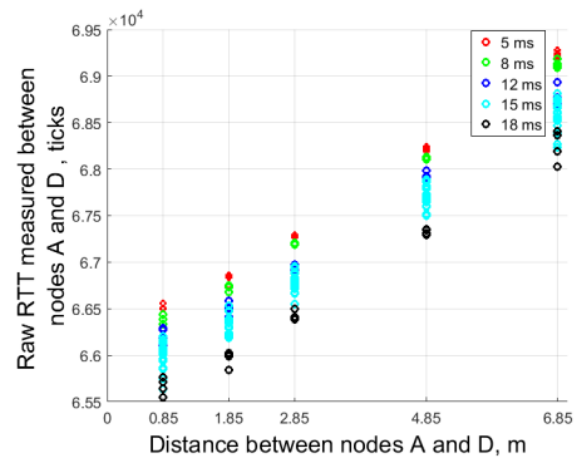

(d) mean RTT for node D measured with basic TWR (RTT1) for the different $\mathrm{T}_{\mathrm{A}}{ }^{\mathrm{RSP}}$ values (mode V0 filtered out)

Fig. 8. Results for mean RTT measured for the calibration runs for basic TWR and

SSA-TWR for static (C) and moved (D) anchors

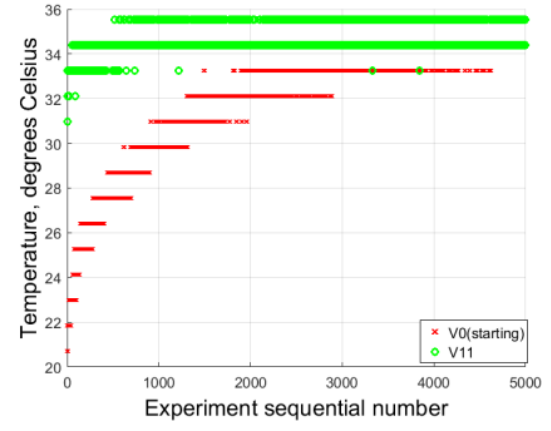

(a) temperature of node $\mathrm{D}$ (as reported by internal sensor)

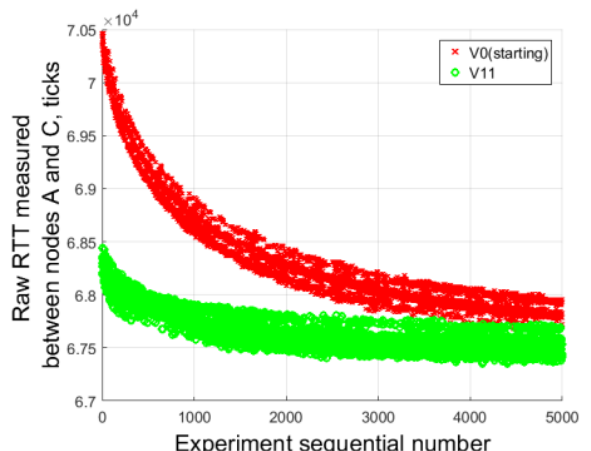

(b) RTT for node D measured with basic TWR (RTT1)

Fig. 9. Illustration of the UWB transceiver's temperature variation for calibration modes V0 and V11 and of its effect on the RTT measured with basic TWR(RTT1).

RTT differed for more than $100 \mathrm{~ns}$. The two possible reasons for this are the effect of the $T_{A}{ }^{R S P}$ (can be seen from Fig. 8(d)) and the individual differences in the antenna delays for the radio transceivers for anchors (refer to [36]).

While analyzing the obtained results we have noted that for all the tested locations of node D, the results for the very first mode (i.e., V0 - marked with the red ' $x$ ' in Figs. 8 (a)-(c)) differed a lot from the other measurements. After analyzing the other collected data we have noticed that the variation of the transceiver's temperature over the experiment run for V0 is the biggest. This is illustrated in Fig. 9 by the temperature profile of the UWB transceiver while running the two calibration modes with identical settings for node D located 4.85 meters apart from the tag (the similar behavior was observed also for the other modes). We expect that the observable effect is caused by the self heat up of the UWB transceiver in the process of its operation. Before the start of the experiment the transceiver is turned off (see Fig.5) and the temperature of the 
chip is close to the room one. Once the experiments start, the transceiver is enabled and starts actively switching between receive and transmit modes, which results in its heat up. As one can clearly see from Fig. 9 (b), with the increase of the temperature from 20 to 35 degrees Celsius, the reported RTT drops from $1100 \mathrm{~ns}$ to about $1060 \mathrm{~ns}$. As can be easily calculated, the difference in the $40 \mathrm{~ns}$ error in RTT gets transformed to the distance error of about 3 meters and thus can dramatically affect the localization accuracy. This observed phenomenon emphasizes the need of accounting the temperature of the radio transceivers in the process of the localization on one hand, and raises the question of whether the heating up of the UWB transceivers should be accounted when designing the localization protocols, as well as the localization-enabled communication protocols on the other hand.

Since the analysis of the results for calibration modes showed the reproducibility of the results and did not reveal any substantial anomalies in the collected data, we proceeded with the analysis of the results for the experimental modes.

\subsection{Analysis of the experimental results}

The statistically processed results of the measurements are presented in Fig. 10. The plots illustrate the mean and the standard deviation over the 5000 measurements for each of the experimental modes and for each position of node D. Since some of the trends cannot be easily detected from the presented chart, we have used the method of least squares to find the linear approximation for the sets of the mode settings and compared the results for these sets. Due to the limited space, we do not present the respective results in the current paper and just report the results coming from analysis. The respective results and the experimental data are planned to be made publicly available from the web page of the research group ${ }^{3}$ by the time of paper's publication.

Before analyzing the results we have to note that the obtained results have two anomalies corresponding to experimental modes 83 (node D at $2.85 \mathrm{~m}$ ) and 132 (node $\mathrm{D}$ at $4.85 \mathrm{~m}$ ). For these the mean RTT appeared to be much lower (512 ns for mode 83 and $980 \mathrm{~ns}$ for mode 132 with basic TWR(RTT1) for node D) and the standard deviation much higher than the values obtained with the other experimental settings. To get a better understanding of these anomalies, in Fig. 11 are plotted the results of the individual RTT tests, for the mode which has produced the anomaly and the following test mode. It is hard to make an accurate conclusion about the reason causing these anomalies, but since the anomaly measurements are characterized with lower RTT, we do not think that the changes in the environment (e.g., an LoS blockade) may have

${ }^{3}$ http://www.cwc.oulu.fi/wnl/ 
caused them. Also the analysis of the temperature of the transceivers did not reveal any substantial differences. Thus, we expect that the anomalies were the result of unexpected behaviour of the radio transceiver. Note that as can be seen from the presented results, the further measurements do not seem to be affected by these anomalies.

The visual analysis of the plots depicted in Figs. 10 (a)-(c) reveals that both the channels used and the data rate strongly affects the measured RTT. Namely, the measurements done with the rate of $110 \mathrm{kbps}$ give the RTT, which is $10-15 \mathrm{~ns}$ higher than the one obtained with $850 \mathrm{kbps}$ rate. The trend seems to be consistent for all the tested channels as well as the other settings. This result seems somewhat strange, given that the transmission and reception of a UWB packet is time stamped at the start of its PHR. We

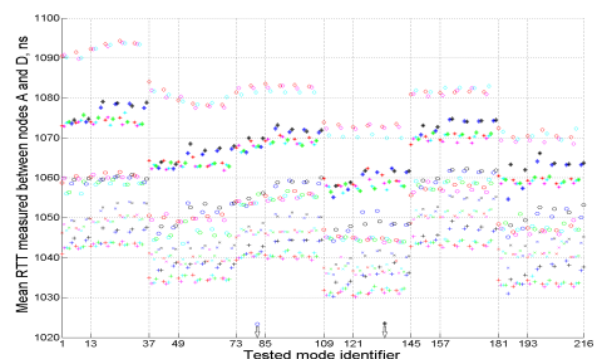

(a) mean RTT for node D measured with basic TWR (RTT1)

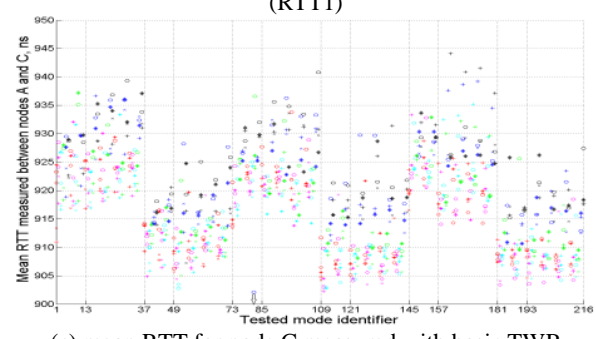

(c) mean RTT for node C measured with basic TWR (RTT1)

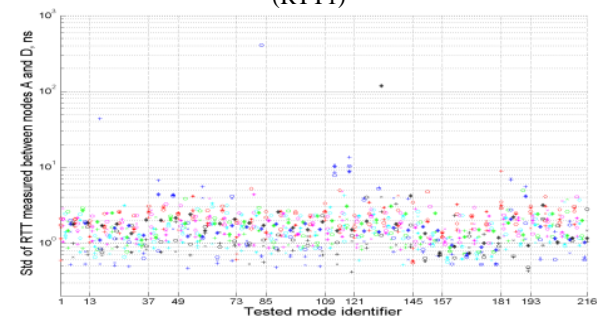

(e) standard deviation of RTT for node D with basic TWR (RTT1)

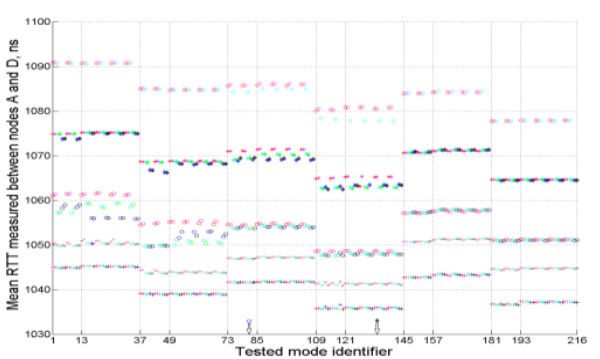

(b) mean RTT for node D measured with SSA-TWR

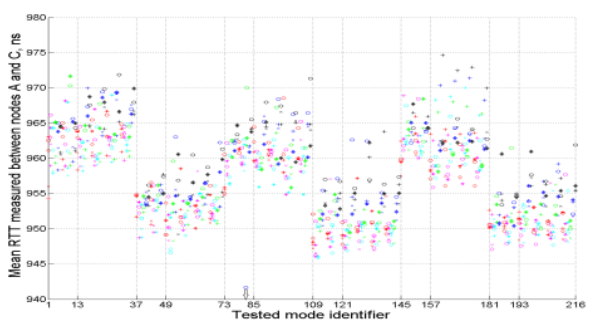

(d) mean RTT for node C measured with SSA-TWR

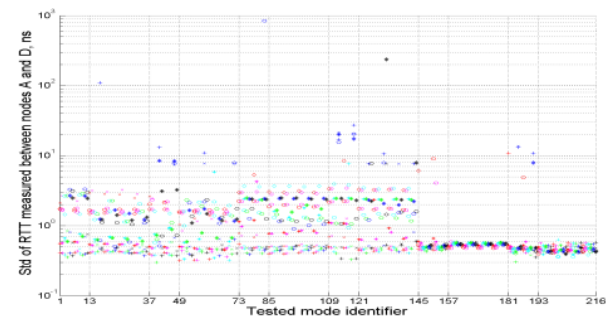

(f) standard deviation of RTT for node D with SSA-TWR 


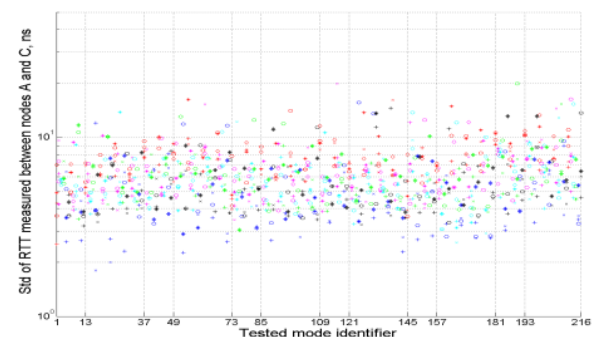

(g) standard deviation of RTT for node $\mathrm{C}$ with basic TWR (RTT1)

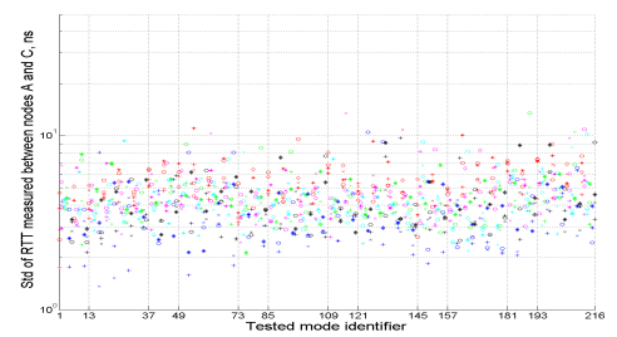

(h) standard deviation of RTT for node C with SSA-TWR

Legend (note that the distance is given between nodes $\mathrm{A}$ and $\mathrm{D}$, the position of node $\mathrm{C}$ was not changed during the experiments) :

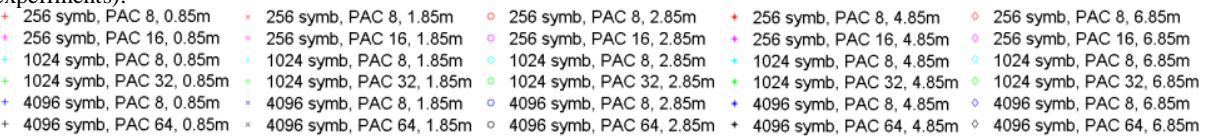
4096 symb, PAC $64,0.85 \mathrm{~m}$ * $4096 \mathrm{symb}$, PAC $64,1.85 \mathrm{~m} \div 4096 \mathrm{symb}$, PAC $64,2.85 \mathrm{~m}+4096 \mathrm{symb}$, PAC $64,4.85 \mathrm{~m} \div 4096 \mathrm{symb}$, PAC $64,6.85 \mathrm{~m}$

Fig. 10. Effect of the parameters on the mean and standard deviation of the RTT

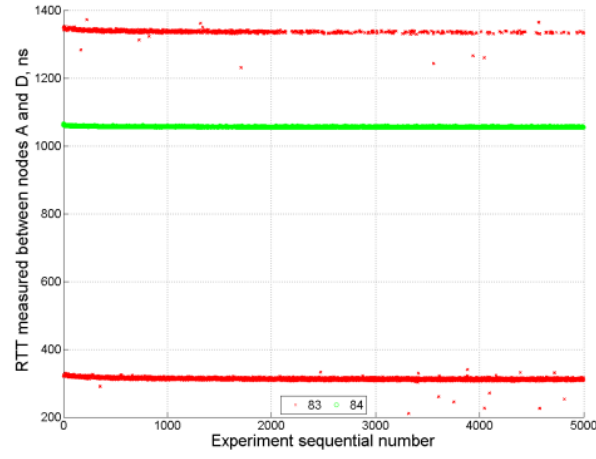

(a) Mode 83, distance between anchor and tag is 2.85

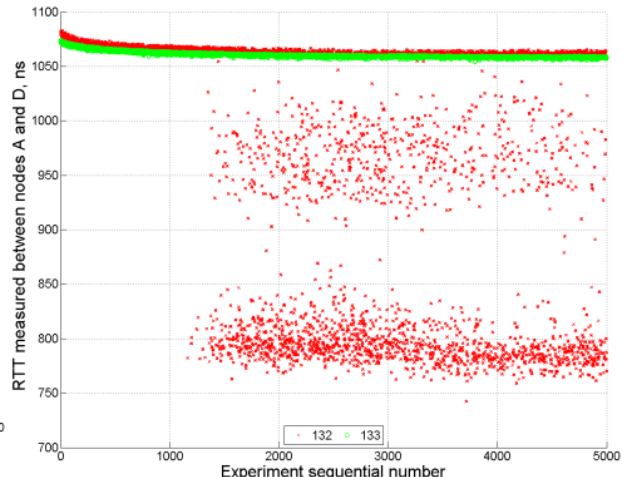

(b) Mode 132, distance between anchor and tag is $4.85 \mathrm{~m}$

Fig. 11. Measured RTT using basic TWR(RTT1) for node D for anomaly points.

had a hypothesis that this effect is caused by the heat up of the tag and analyzed the node's temperature profiles in the different modes differing in rate and PRF values. Even though for some of them we have observed the difference in the tag's temperature, for many others the reported temperature was about the same. This makes us draw the conclusion that the temperature and the heat up of the tag (e.g., due to spending more time for sending or receiving a packet) is not the only factor causing the observed behavior. Out of the three tested channels, channel 2 tends to give slightly higher RTT results than the two other tested channels. Interestingly, the difference seems to increase with the increase of the distance between the tag and the anchor. In respect to the preamble length for basic TWR, the shorter preambles tend to give the lower overall RTT. The trends related to the PAC are not obvious. E.g., for 1024 and 4096 symbol preambles, the use of higher PAC resulted in getting slightly higher RTT measurements than the ones obtained with $\mathrm{PAC}=8$. For 256 symbol preamble, the results are opposite. The RTT obtained with the lower PRF appears to be slightly lower than the one got 
with higher PRF, but the difference is very minor. No significant effect of the used preamble code was observed.

The effect of the different communication mode parameters on the standard deviation of the measurements can be assessed from Figs. 10 (e)-(f). Note that the charts have vertical axis in logarithmic scale. As can be noted from the presented results, for the static anchor the deviation of the measurements appears to be about the same for all the three tested channels. In contrast to this, for the SSA-TWR and the anchor node, which was moved, the tests done in channel $5(6489.6 \mathrm{MHz}$ central frequency and $499.2 \mathrm{MHz}$ bandwidth) showed much lower deviation of the measurements (especially for $\mathrm{PRF}=64$ $\mathrm{MHz}$ ). Another interesting point is that unlike the measurements done with the other frequency channels, the standard deviation of the results obtained with channel 5 do not seem to change much with the distance between the tag and the anchor. Unfortunately, we cannot provide any reasonable explanation for this behavior based on the results of the conducted measurements. In respect to the effect of the other parameters, it can be noted that for the static anchor the use of the longest preambles often resulted in a lower deviation of the RTT measurements. Nonetheless, for the anchor D the use of long preambles (especially with $\mathrm{PRF}=16 \mathrm{MHz}$ ) often resulted in very high deviations of the measurements. Unfortunately, the conducted analysis did not allow us to reveal clearly the effects of the other parameters (i.e., rate, PAC, used preamble code) on the deviation of the RTT.

Finally, we have used the obtained results to estimate the actual distance measurement error. For this, we have first constructed (using the method of least squares) the linear approximation for the dependence of the mean RTT from the distance for each single tested mode having the linear slope in $\mathrm{m}^{-1}$ given by

$$
\text { slope }=(1 / c) / T=213.14
$$

where $c$ is the speed of light in $\mathrm{m} / \mathrm{s}$ and $T=1 /\left(128 \cdot 499.2 \cdot 10^{6}\right)$ is the resolution of the time stamp for the used UWB transceiver in ticks per second. Next, for each of the 

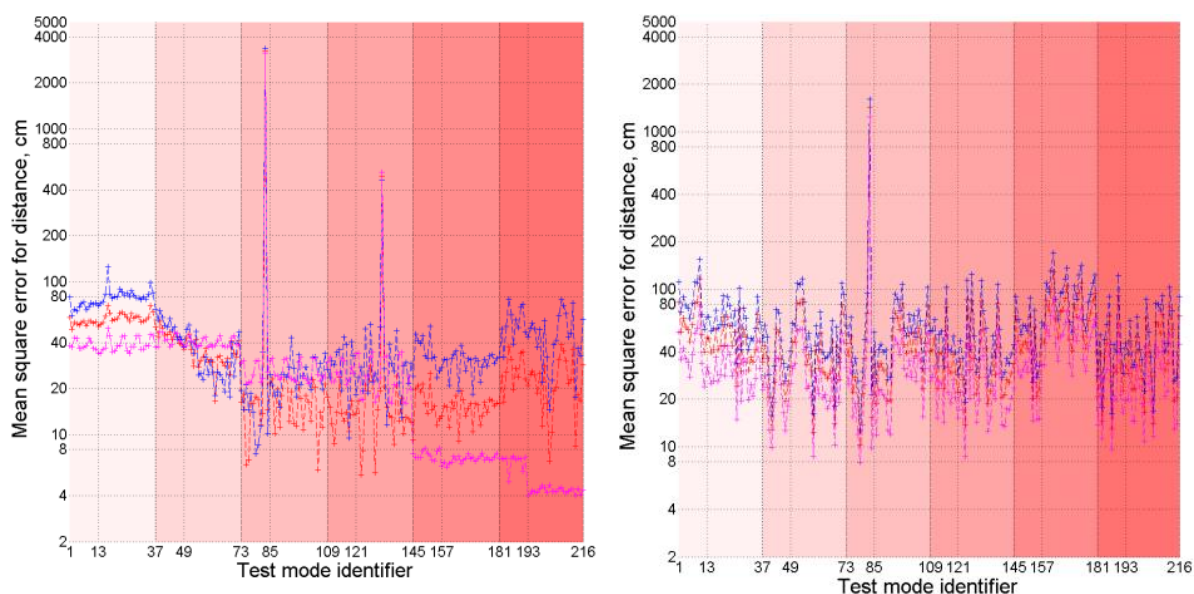

(a) Average accuracy of distance measurement between

(b) Average accuracy of distance measurement between nodes nodes $\mathrm{A}$ and $\mathrm{D}$ $\mathrm{A}$ and $\mathrm{C}$

Legend:

Ch2 110kbs C. Ch2 850kbps Ch4 110kbps Ch4 850kbp I. Ch5 110kbps I Ch5 850kbps

-+-basic TWR(RTT1) - ---basic TWR(RTT2) - -+-SSA-TWR

Fig. 12. Mean localization error for the tested modes.

tested modes we have calculated the root mean square error (RMSE) between the reference measurement with the laser distance meter and the distance obtained using from RTT. The described procedure was executed for the both anchor nodes and the three protocols, namely the two basic TWR (using RTT1 and RTT2 responses) and the SSA-TWR. The results are summarized in Fig. 12.

As can be seen, for the distance measured between the tag and anchor $\mathrm{D}$, the minimum RMSE of $4 \mathrm{~cm}$ is obtained with SSA-TWR when operating in channel 5 with $850 \mathrm{kbps}$ rate and PRF=64 MHz. The use of other channels resulted in much higher RMSE values (over $20 \mathrm{~cm}$ for channel 4 and about $40 \mathrm{~cm}$ for channel 4). What is more than this, while using PRF $=64 \mathrm{MHz}$, data rate of $850 \mathrm{kbps}$ and operating in channel 2, as well as while working in channel 4, the RMSE of SSA-TWR even appeared to be higher than the one for basic TWR based on the first reply. The minimum RMSE of $8 \mathrm{~cm}$ for the distance estimated between anchor $\mathrm{C}$ and the tag was obtained with SSA-TWR while using mode 79 (channel 4, $110 \mathrm{kbps}$ rate, 256 symbol preamble, $\mathrm{PAC}=8, \mathrm{PRF}=16 \mathrm{MHz}$, preamble code 2). As can be seen from the presented results, for the majority of the tests the performance of SSA-TWR was superior to the one of basic TWR. Namely, for static node the SSA-TWR gave 33\% lower average error, for mobile node the average error for SSA-TWR was lower more than 2 times.

\section{Conclusions and lessons learned.}


In this study we address the problem of enabling accurate UWB-based wireless indoor localization of machines and humans. The previous work of us enabling a single-sided asymmetric TWR (SSA-TWR) protocol intended for the asymmetric scenario, when an advanced but power limited tag needs to determine its position in respect to more simple but non energy-constrained anchors, have revealed that the accuracy of the UWB ranging is strongly affected by the communication settings. Due to this reason, in this work by the means of empirical measurements we investigated how the various communication mode parameters affect the performance of the UWB localization in practice. Using the real-life the IEEE 802.15.4-2015 UWB enabled radio transceivers we have conducted an extensive measurement campaign lasting over 200 hours almost nonstop and involving transmission of more than 30 million ranging packets. In total we have tested over 200 different operation modes by varying seven different communication and localization protocol parameters. The results of the measurement have been statistically processed and further analyzed to determine the possible dependences.

The obtained results clearly show that the accuracy of real-life RTT measurements are affected by several communication mode parameters. Namely, we have shown that the accuracy of ranging strongly depends on the used channel, data rate and pulse repetition frequency. The other tested parameters such as the preamble duration, preamble acquisition chunk and preamble code had less notable effect. What is more than this, we have shown that the compensation value which should be subtracted from the RTT to compensate, e.g., for the antenna delays, is also affected by the communication mode configuration. In respect to the parameters of the localization protocol, we have noted the strong negative effect the increase of the delay between the reception of the localization request and the transmission of the localization response for all the tested localization protocols had. Given that the response delay depends on the used communication mode settings (especially the data rate and the length of the preamble), which, in their turn, affect the communication range and the packet error rate of the UWB communication (as shown, e.g., in [29]), the optimal selection of these parameters is often non-trivial. Finally, the conducted experiments demonstrated the devastating effect which self-heating of the UWB transceiver in the process of its operation may have on the RTT measurements in the real-life systems. Note that in our experiments the UWB transceivers were located off the human body and were installed on sufficiently big printed circuitry boards (PCB) acting also as a heatsink. The operation of the transceiver close to a human body (e.g., in BANs) may make the negative consequences even more notable. This emphasizes the importance of monitoring the transceiver's temperature and developing the efficient methods for compensating for the error caused by temperature variations. Aside of this, we consider that the noted effect may even need to be taken into account while developing the upper layers of the 
communication stack (i.e., link and network layers) in order to facilitate the temperature-neutral operation of a transceiver.

Even though in our experiments we have obtained the localization accuracy of below $10 \mathrm{~cm}$ with the IEEE 802.15.4-2015 HRP compatible transceivers, by no means this should be treated as the ultimate performance limit of the devices used. We would like to note that the measurements have been conducted having the sufficiently long response delay (typically $-15 \mathrm{~ms}$ ), which can be reduced (at least for some operation modes) to under $1 \mathrm{~ms}$. Next, we have not attempted to compensate or account for the temperature drift in the process of our experiments. What is more than this, since prior to the experiments we have underestimated this effect, the measurements were done in a room with no climate control, and thus, the variation of the room temperature may have impacted the results of our measurements. Finally, in our experiments we have tested only a portion of all the possible operation modes. Nonetheless, the presented results clearly show that the effect of the communication mode parameters on the performance of the UWB localization should not be underestimated and the respective studies, using both analytic and experimental methods, have to be continued. Another important problem still to be studied is the characterization of environment's effect (e.g., in respect to various rooms) on the UWB localization as well as the optimal selection of the localization parameters based on the available information about the environment of operation. 


\section{References}

1 B.R. Decastro et al., "Modeling time-location patterns of inner-city high school students in New York and Los Angeles using a longitudinal approach with generalized estimating equations”, J. Expos. Sci. Environ. Epidemiol, vol. 17, pp. 233-247, May 2006.

2 D. Lymberopoulos et al., "A Realistic Evaluation and Comparison of Indoor Location Technologies: Experiences and Lessons Learned," in Proc. 14th Int. Conf. Inf. Proc. Sensor Netw., Seattle, USA, 2015, pp. 178-189.

3 D. Dardari, P. Closas and P.M. Djuric, "Indoor Tracking: Theory, Methods, and Technologies," IEEE Trans. Vehic. Tech., vol. 64, no. 4, pp. 1263-1278, Feb. 2015.

4 A. Georgiev and P. K. Allen, "Localization methods for a mobile robot in urban environments," IEEE Trans. Robotics, vol. 20, no. 5, pp. 851-864, Oct. 2004.

5 Jiang Xiao et al., "A Survey on Wireless Indoor Localization from the Device Perspective," ACM Comput. Surv. vol. 49, no. 2, article 25, June 2016.

6 I. Oppermann, M. Hämäläinen and J. Iinatti (Eds.), UWB Theory and Applications, John Wiley \& Sons, Chichester, UK, 2004.

7 D. Dardari et al., "Ranging With Ultrawide Bandwidth Signals in Multipath Environments," Proc. IEEE, vol. 97, no. 2, pp. 404-426, Feb. 2009.

8 D. Lymberopoulos et al., "2016 Microsoft Indoor Localization Competition", available: https://www.microsoft.com/en-us/research/wpcontent/uploads/2015/10/2016_indoorloc_competition.pptx

9 A. Alarifi et al., "UltraWideband Indoor Positioning Technologies: Analysis and Recent Advances”, Sensors, vol. 16, no. 5, pp.707, May 2016.

10 Yunhao Liu and Zheng Yang, Location, Localization, and Localizability: Locationawareness Technology for Wireless Networks, Springer, New York, USA, 2011.

11 Yunhao Liu et al., "Location, localization, and localizability", J. Computer Science Tech., vol. 25, no. 2, pp. 274-297, Mar. 2010

12 "IEEE Standard for Low-Rate Wireless Networks," in IEEE Std. 802.15.4-2015 (Revision of IEEE Std 802.15.4-2011), pp.1-709, April 222016.

13 H. Kim, "Double-sided two-way ranging algorithm to reduce ranging time," IEEE Commun. Letters, vol. 13, no. 7, pp. 486-488, July 2009.

14 M. Kwak and J. Chong, "A new Double Two-Way Ranging algorithm for ranging system," in Proc. 2nd IEEE Int. Conf. Netw. Infrastructure Digital Content, Beijing, China, 2010, pp. 470-473.

15 R. Dalce, A. van den Bossche and T. Val, "Indoor Self-Localization in a WSN, based on Time Of Flight: Propositions and Demonstrator", in Proc. Int. Conf. Indoor Positionning Indoor Navigation, Montbeliard, France, 2013, pp. 105-110.

16 R. Dalce, A. van den Bossche and T. Val, "Reducing Localisation Overhead: A Ranging Protocol and an Enhanced Algorithm for UWB-Based WSNs", in Proc. $81^{\text {st }}$ IEEE Vehicular Technology Conf. (VTC Spring), Glasgow, Scotland, 2015, pp. 1-5, 2015.

17 R. Dalce, A. van den Bossche and T. Val, "An experimental performance study of an original ranging protocol based on an IEEE 802.15.4a UWB testbed," in Proc. IEEE Int. Conf. UltraWideBand, Paris, France, 2014, pp. 7-12.

18 B. Kempke et al., "Demo: PolyPoint: High-Precision Indoor Localization with UWB," in Proc. 13th ACM Conf. Embedded Netw. Sensor Syst, Seoul, ROK, 2015, pp. 483-484.

19 M. Simek et al., "Evaluation of PHY layer throughput of ultra wide band IEEE 802.15.4a technology," in Proc. $36^{\text {th }}$ Int. Conf. Telecom. Signal Proc., Rome, Italy, 2013, pp. 105-110.

20 N. Ullah et al., "Throughput limits of UWB based 802.15.4a," in Proc. Int. Conf. Inf. Commun. Tech. Convergence, Jeju, ROK, 2010, pp. 166-167.

21 T. Gigl et al., "Ranging performance of the IEEE 802.15.4a UWB standard under FCC/CEPT regulations", J. Electrical Computer Eng., vol. 2012, article 218930. 
22 V. Niemelä et al., "Performance of IEEE 802.15.4a UWB WBAN Receivers in a Real Hospital Environment," in Proc. 4th Int. Symp. Medical Inf. Commun. Tech, Taipei, Taiwan, 2010, pp. 1-4.

23 F. Erlacher et al., "AvaRange - Using Sensor Network Ranging Techniques to Explore the Dynamics of Avalanches," in Proc. $12^{\text {th }}$ Wireless On-demand Netw. Syst. Services Conf., Cortina d'Ampezzo, Italy, 2016, pp. 120-123.

24 B. Kempke, P. Pannuto and P. Dutta, "PolyPoint: Guiding Indoor Quadrotors with UltraWideband Localization," in Proc. 2nd Int. Workshop Hot Topics Wireless, New York, NY, USA, 2015, pp. 16-20.

25 B. Silva et al., "Positioning infrastructure for industrial automation systems based on UWB wireless communication," in Proc. 40th Annu. Conf. IEEE Industrial Electronics Soc., Dallas, TX, 2014, pp. 3919-3925.

26 J. Wang, A. K. Raja and Z. Pang, "Prototyping and Experimental Comparison of IR-UWB Based High Precision Localization Technologies," in Proc. IEEE 12th Int. Conf. Ubiquitous Intelligence Comput., Beijing, China, 2015, pp. 1187-1192.

27 F. Hammer et al., "Performance Evaluation of 3D-Position Estimation Systems," IEEE Sensors J., vol. 16, no. 16, pp. 6416-6424, Aug. 2016.

28 W. Chantaweesomboon et al., "On performance study of UWB real time locating system," in Proc. 7th Int. Conf. Inf. Commun. Tech. Embedded Syst., Bangkok, Thailand, 2016, pp. 19-24.

$29 \mathrm{Ke} \mathrm{Xu}$, Performance measurements of DW1000 implementing IEEE standard 802.15.42011 impulse radio ultra-wideband technology, M.Sc. thesis, University of Oulu, 2016.

30 "IEEE Standard for Information Technology - Telecommunications and Information Exchange Between Systems - Local and Metropolitan Area Networks - Specific Requirement Part 15.4: Wireless Medium Access Control (MAC) and Physical Layer (PHY) Specifications for Low-Rate Wireless Personal Area Networks (WPANs)," IEEE Std 802.15.4a-2007 (Amendment to IEEE Std. 802.15.4-2006), pp.1-203, 2007.

31 "IEEE Standard for Local and Metropolitan Area Networks-Part 15.4: Low-Rate Wireless Personal Area Networks (LR-WPANs)," in IEEE Std. 802.15.4-2011 (Revision of IEEE Std 802.15.4-2006), pp.1-314, Sept. 52011.

32 R. Cardinal et al., "UWB Ranging Accuracy in High- and Low-Data-Rate Applications", IEEE Trans. Microwave Theory Tech., vol. 54, no. 4, pp. 1865-1875, Apr. 2006.

33 Woo Cheol Chung and Dong Sam Ha, "An accurate ultra wideband (UWB) ranging for precision asset location", in Proc. IEEE Conf. Ultra Wideband Syst. Tech., Reston, Virginia, 2003, pp. 389-393.

34 I. Guvenc et al., "TOA Estimation for IR-UWB Systems With Different Transceiver Types," IEEE Trans. Microwave Theory Tech., vol. 54, no. 4, pp. 1876-1886, Apr. 2006.

35 K. Mikhaylov et al., "On the Selection of Protocol and Parameters for UWB-based Wireless Indoors Localization", in Proc. 10th Int. Symp. Medical Inf. Commun. Tech., Worcester, USA, 2016, pp. 1-5.

36 K. Mikhaylov et al., "Extensible Modular Wireless Sensor and Actuator Network and IoT Platform with Plug\&Play Module Connection", in Proc. ACM/IEEE Int. Conf. Inf. Proc. Sensor Netw., Seattle, USA, 2015, pp. 1-5.

37 K. Mikhaylov and J. Petäjäjärvi, "Design and Implementation of the Plug\&Play enabled Flexible Modular Wireless Sensor and Actuator Network Platform," in press.

38 DecaWave, ScenSor DWM1000 Module, available: http://www.decawave.com/products/dwm1000-module

39 DecaWave, EVK1000 user manual, available: http://www.decawave.com/sites/default/files/product-pdf/evk1000_user_manual_v107.pdf 